\title{
Identification through movement analysis of chronic low back pain pathological spinal movements patterns and their sensibility to change during exercise
}

Stefano Negrini ${ }^{1,2}$, Barbara Piovanelli $^{1}$, Cinzia Amici $^{2}$, Sabrina Donzelli ${ }^{3}$, Fabio Zaina ${ }^{3}$

1. IRCCS Fondazione Don Carlo Gnocchi, Milano, Milano, ITALY, Italy

2. University of Brescia, Clinical and Experimental Sciences Department, Brescia, Italy

3. ISICO, Italian Scientific Spine Institute, Milan, Italy, Italy

INTRODUCTION: Chronic low back pain (CLBP) is a bio-psycho-social syndrome causing disability. While there are multiple imaging systems for the anatomical structures, in vivo movement evaluation has scarcely been performed. ${ }^{1}$ Our aim was to pilot in a CLBP population a movement analysis protocol developed in healthy participants ${ }^{2}$, and verify correlations with pain, disability and treatment effects.

METHODS: We recruited a convenience sample of 10 CLBP patients who were referred for a group exercise therapy (strengthening, core stability, active stretching and postural re-education) ${ }^{3,4}$ We used a noninvasive optoelectronic full spine evaluation according to a previously developed protocol ${ }^{2}$. We analyzed anterior flexion, lateral bending and rotation movements, and collected Numerical Rating (NRS) and Oswestry (ODI) scales before and after treatment. We performed a qualitative analysis to identify possible abnormal movement patterns, that have been quantified through 4 points Likert scales: their interobservers repeatability has been checked comparing three operators ( 1 expert and 2 students). A total score for each movement has then been calculated as well as its variations with treatment, and correlations with ODI and NRS.

RESULTS: Comparing normal to pathological patterns (fig 1) we identified the following parameters: for all movements total Range of Motion, fluidity, symmetry, and pelvis center; we also added for lateral bending the lumbopelvic rhythm, for flexion and rotation the arrival overdrive, and for flexion the altered Anticipatory Postural Adjustments. Inter-operators repeatability resulted in a Cohen k $0.21-0.4$ in $13 \%$ of parameters, $0.41-0.6$ in 47\%, 0.61-0.8 in 32\% and 0.81-1 in 9\%. With treatment, ODI and NRS improved and the total score decreased in all movements: flexion $(p<0.01)$, bending $(p<0.01)$ and rotation $(p<0.0005)$. We did not find any correlation with ODI and NRS.

DISCUSSION: In this study we have identified some reliable qualitative patterns of pathological movement in CLBP that showed to be sensible to treatment, even if not correlated to subjective scales like ODI and NRS. Future studies should check these preliminary results in wider populations and different treatments, while a quantification of these qualitative parameters is under development.

1. Negrini, S. et al. Trunk motion analysis: a systematic review from a clinical and methodological perspective. Eur. J. Phys. Rehabil. Med. 52, 583-592 (2016)

2. Barbara, P. et al. Functional assessment of the spine through an optoelectronic system. Gait \&amp; Posture Volume 57, Supplement 1, Pages 347-348 (2017)

3. Sahin, N. et al. Effectiveness of back school for treatment of pain and functional disability in patients with chronic low back pain: a randomized controlled trial. J. Rehabil. Med. 43, 224-229 (2011)

4. Pfingsten, M. et al. Multidisciplinary treatment program on chronic low back pain, part 3. Psychosocial aspects. Schmerz Berl. Ger. 10, 326-344 (1996) 